\title{
AUTISM SPECTRUM DISORDER IN A CHILD WITH TUBEROUS SCLEROSIS: WHERE IS THE NEUROBIOLOGICAL LINK?
}

\author{
Rajeev Ranjan ${ }^{1}$, Santanu Nath ${ }^{2}$, Meha Jain ${ }^{1} \&$ Pankaj Kumar ${ }^{1}$ \\ ${ }^{1}$ Department of Psychiatry, All India Institute of Medical Sciences (AIIMS), Patna, Bihar, India \\ ${ }^{2}$ Department of Psychiatry, All India Institute of Medical Sciences (AIIMS), Deoghar, Jharkhand, India
}

received: 4.1.2021;

revised: 3.3.2021;

accepted: 15.3 .2021

\section{INTRODUCTION}

Autism spectrum disorders (ASD) are neurodevelopmental disorders that manifests in children with poor social and communication skills, repetitive behavioural repertoire and occasionally with language problems. Tuberous sclerosis complex (TSC) is a genetic disorder characterized by hamartomas in several organs. The association of ASD and TS is not unknown. Infact, TS is considered to be the most common genetic cause for ASD (Curatolo et al. 2010). There are multiple factors specific for TS which are possible predictors for the development of ASD (Mitchell et al. 2017). We hereby report a case of a boy suffering from ASD who was incidentally diagnosed with TS while discussing the management and the possible neurobiological underpinnings behind this association.

\section{CASE PRESENTATION}

A 6 year old boy having a past history of seizures and a family history of seizure disorder and skin lesions in his brother, presented with four years history of insidious onset and a progressive course of symptoms, characterized by speech and language regression, poor social communication, repetitive unusual behaviour (running around bed, spinning inanimate objects with his hands, stacking toys and shoes) with behaviours like smelling food before eating, running away from the hooting of the pressure cooker etc along with hyperactivity and inattention. He also has daily early morning myoclonic jerks and occasional left focal to generalized seizures ever since he was 6 months old till the date of presentation. He started to attend a Kindergarten school for last 6 months and is found to be falling behind his peers in various activities apart from learning the basic alphabets.

On physical examination, white macules were seen over lower abdomen and back and acneform eruptions were seen on his face; more around the nose. On mental status examination, he had poor eye contact, was making an unintelligible sound continuously, did not respond to any calls, didn't talk nor looked at a toy being placed near him. His audiometry (BERA) showed hearing ability within normal limit. A dermatologist opined his skin lesions to be 'ash-leaf spots' in the above mentioned areas along with adenoma sebaceum on his face. Computed Tomography (CT) of Brain showed multiple calcified sub-ependymal nodules seen along bilateral lateral ventricles (Figure 1) suggestive of tuberous sclerosis. A provisional diagnosis of ASD with tuberous sclerosis was made. He was prescribed syrup valproate $(200 \mathrm{mg} / 5 \mathrm{ml}) 5 \mathrm{ml} \mathrm{BD}$ for seizures and syrup risperidone $0.5 \mathrm{ml}$ for his hyperactivity. He was also started on speech and occupational therapy. Over few months he showed improvement in activities of daily living, sensory issues and undesired repetitive behaviour while his social communication continued to be a challenging issue.

\section{DISCUSSION}

The boy with ASD was also incidentally diagnosed to have TS considering his concomitant seizure history, the characteristic skin lesions, a positive family history along with contributory neuroimaging findings. TSC manifests with an array of neuropsychiatric features, called TSC-associated neuropsychiatric disorders (TAND) that includes seizures, behavioural problems, intellectual deficits, ASD, psychiatric disorders like anxiety, depression and psychosis, scholastic difficulties and cognitive deficits (Northrup \& Krueger 2013). ASD is reported in around $16-55 \%$ of those with TSC (Mitchell et al. 2017). It is evident in TSC even within the first 18 months of life when an infant shows a deficit in play, social interaction and reciprocity along with a poor eye contact (Jeste et al. 2008). This is followed by problems like overactivity and stereotypies which becomes evident after the age of 2 years (Curatolo et al. 2010).

The predictors for development of ASD in TS includes early onset of poorly controlled seizures, associated cognitive impairment, epileptiform activity in key brain areas like the temporal lobes and cerebellum on electroencephalogram, presence of cortical tubers in strategic locations viz temporal, frontal lobes and cerebellum, tuber brain proportion and associated white matter abnormalities (Curatolo et al. 2010, Mitchell et al. 2017). 


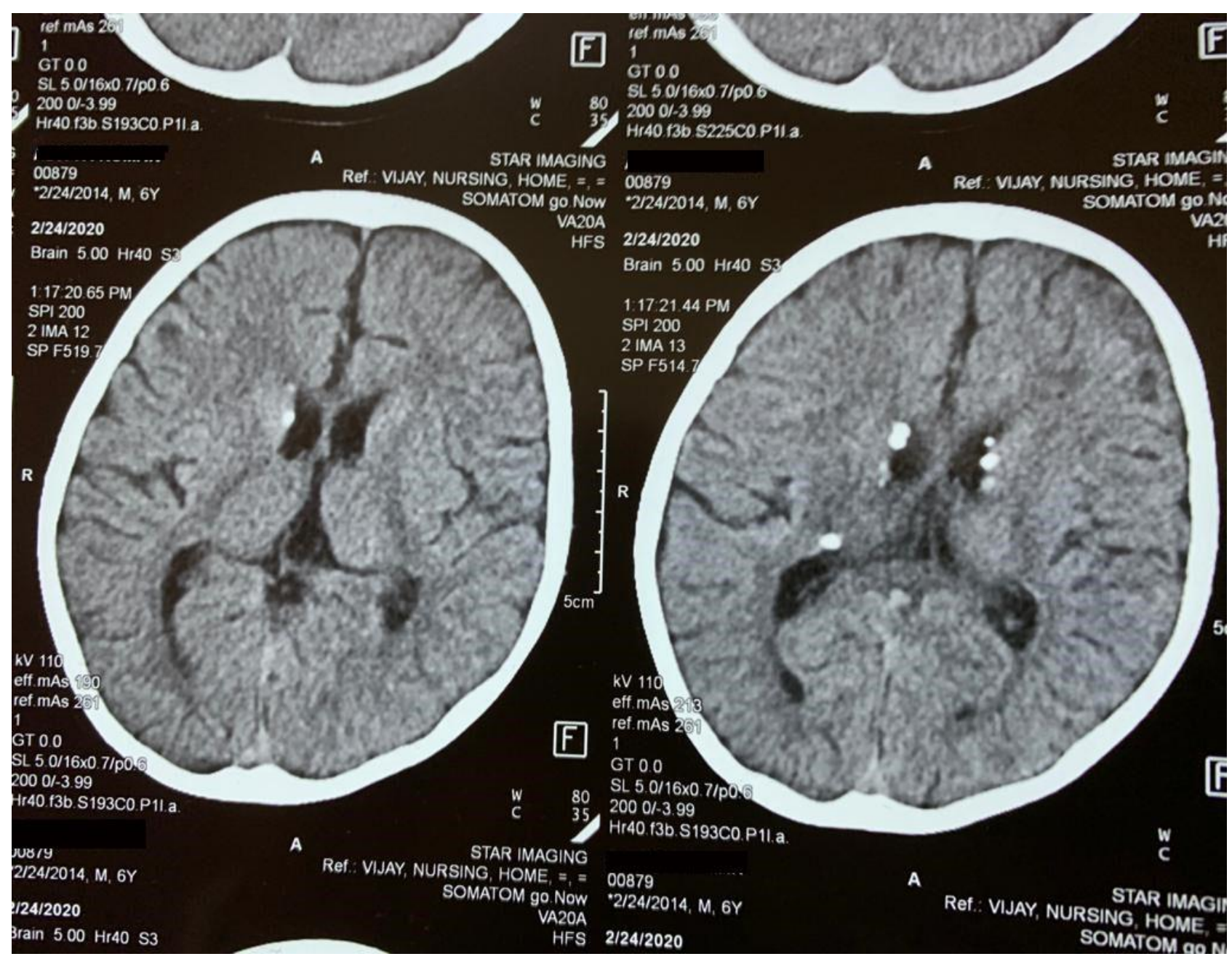

Figure 1. Tubers in sub-ependymal location seen along bilateral lateral ventricles in CT Scan brain

They have also been investigated to have glucose hypometabolism in bilateral lateral temporal cortex, glucose hypermetabolism in the deep cerebellar nuclei and augmented alpha-methyl-tryptophan uptake in the caudate nuclei, which are associated with both stereotypic behaviour and poor social communication (Eluvathingal et al. 2006).

The biological pathways that connect TS with ASD commences from putative TSC mutations which leads to activation of the mTOR (mammalian target of rapamycin), leading to abnormal synaptic functioning, white matter connectivity problems, a misbalance between the excitatory glutamatergic and inhibitory GABA-ergic neurotransmitter function, that has been postulated to ultimately lead to ASD. This mutation also strongly mediates the link between early onset seizures in TS and the development of cognitive deficits and ASD (Curatolo et al. 2010). Early management of seizures with Vigabatrin has been proposed to lessen the neurobehavioural outcomes in TSC (eg ASD) but does not guarantee a favourable outcome in TS children (Bombardieri et al. 2010).

From a management point of view, ASD needs a multispecialist approach that involves psychiatrists, paediatricians, clinical psychologists, occupational and speech therapists. Psychotropics are sometimes warranted as like in our case to manage problematic behaviour and hyperactivity. Few drugs like rapamycin, temsirolimus, deforolimus and everolimus are currently in clinical trials for a variety of indications, including autism occurring with TSC (Sundberg \& Sahin 2015).

We conclude that the case described, highlights the association of ASD with TS which emphasizes the need to evaluate a child suffering from ASD for possible TS when concomitant seizures and neurocutaneous markers are also present. A well concerted management of seizures and also of the autism is warranted with a focus on other systemic manifestations of TS for a holistic management.

\section{Acknowledgements: None.}

Conflict of interest: None to declare.

\section{Contribution of individual authors:}

All authors made equal contribution to this case report in terms of drafting, writing, obtaining the patient' $s$ consent, and revising the paper. 


\section{References}

1. Bombardieri R, Pinci M, Moavero R, Cerminara C, Curatolo P. Early control of seizures improves long-term outcome in children with tuberous sclerosis complex. Eur J Paediatr Neurol 2010; 14:146-149

2. Curatolo P, Napolioni V, Moavero R. Autism spectrum disorders in tuberous sclerosis: pathogenetic pathways and implications for treatment. J Child Neurol 2010; $25: 873-80$

3. Eluvathingal TJ, Behen ME, Chugani HT, Janisse J, Bernardi B, Chakraborty P, et al. Cerebellar lesions in tuberous sclerosis complex: neurobehavioral and neuroimaging correlates. J Child Neurol 2006;21:846-851

4. Jeste SS, Sahin M, Bolton P, Ploubidis GB, Humphrey A.
Characterization of autism in young children with tuberous sclerosis complex. J Child Neurol 2008; 23:520-525

5. Mitchell R, Barton S, Harvey AS, Williams K. Risk factors for the development of autism spectrum disorder in children with tuberous sclerosis complex: protocol for a systematic review. Syst Rev 2017; 6:49

6. Northrup H, Krueger DA; International Tuberous Sclerosis Complex Consensus Group. Tuberous sclerosis complex diagnostic criteria update: recommendations of the 2012 Iinternational Tuberous Sclerosis Complex Consensus Conference. Pediatr Neurol 2013; 49:243-254

7. Sundberg M, Sahin M. Cerebellar Development and Autism Spectrum Disorder in Tuberous Sclerosis Complex. J Child Neurol. 2015; 30:1954-1962

Correspondence:

Assistant Professor Santanu Nath, MBBS, MD, DNB

Department of Psychiatry, All India Institute of Medical Sciences (AIIMS)

Deoghar, PTI Campus, Daburgram, District: Deoghar, PIN: 814142, Jharkhand, India

E-mail: beta.santanu@gmail.com 